\title{
New Approach to Assess Unbalance and Harmonic Distortion in Power Systems
}

\author{
Patricio Salmerón, Alejandro Pérez, Salvador P. Litrán \\ Department of Electrical Engineering \\ Escuela Técnica Superior de Ingeniería \\ University of Huelva \\ Campus of La Rábida - Palos de la Frontera, 21819 Huelva (Spain) \\ Phone/Fax number:+0034 959217583/959217304 \\ E-mail: patricio@uhu.es, aperez@uhu.es, salvador@uhu.es
}

\begin{abstract}
This paper presents a new decomposition of the apparent power for power systems in unbalance conditions and harmonic distortion at Std. 1459 framework. From the new terms of power defined, a set of appropriate indicators to assess the unbalance in terms of harmonic distortion are introduced. From here, we obtain the balanced and unbalanced components for each set of voltage and current harmonics. The process is carried out through the application of the FFT to the Park vectors of voltage and current. This allows to develop an efficient algorithm from the computational point of view.. The algorithm has been implemented in a virtual instrument for measurements of a practical case. The results obtained show the utility of the proposed approach to assess the unbalance in the presence of distortion.
\end{abstract}

\section{Key words}

Power quality, harmonics, unbalance, measurement.

\section{Introduction}

The ideal operating conditions of a power system correspond to a three-phase system with sinusoidal waveforms of voltage and current according to a balanced arrangement. However, the different operating conditions of the system results in an unbalanced system with distorted waveforms due to the presence of non-linear loads; as a consequence serious problems of EPQ (Electric Power Quality) are created [1]. While the problems associated with the presence of harmonics have been analyzed with certain profusion in recent years, the characterization of unbalance in power systems has been less attention.

Among the main effects derived from the presence of unbalance, here identifies the following. First, the three phase transformers connected in Dy with the purpose of avoiding the penetration of zero sequence components, do not prove effective as a result of unbalanced of the harmonic components multiples of three. In this case, the transformer loses its status as the third harmonic filter. Secondly, the control circuits of power converters that dependent on the crossing by zero of the supposed balanced supply voltage, are subjected to malfunction when the system is unbalanced. Finally, in many situations the presence of a small imbalance in the voltages may produce an unexpected increase of line currents. Other effects could be enumerated. This justifies the need to provide an adequate characterization of the unbalance in conditions of harmonic distortion [24].

The Std 1459 presents a decomposition of the apparent power as a term of fundamental apparent power and other term of not fundamental apparent power, [5]. The direct sequence power and unbalance power are distinguished in fundamental apparent power term. In the second are includes three components associated to the distortion. In this way the unbalanced is characterized by unbalance measured at the fundamental frequency. However, the presence of unbalanced is found in all the harmonics present in the system. Therefore, an adequate characterization of the unbalance in nonsinusoidal conditions requires a review of the decomposition of power established on the standard and the index associated with it. This work has been a re-formulation of the apparent power according to the same approach adopted in the standard but which appears both, the imbalance of the fundamental harmonic, as the imbalance of all harmonics present in the system. For this, determines the phasors of symmetrical components of each harmonic of the voltage and current through the use of the FFT Park vectors. Thus, may be distinguished in a practical way direct sequence and inverse sequence components of each harmonic. The homopolar sequence components of each harmonic are obtained through a supplementary estimate, [6].

Finally, the proposed algorithm has been implemented through a virtual instrument, VI, based on DSP cards. The VI has been used for measurement of new quality 
indices defined in a three-phase unbalanced and distorted system. The system consisting of three-phase linear/non linear, balanced/unbalanced loads has allowed demonstrate the applicability of the proposal.

\section{Voltage and Current Components in Unbalance and Nonsinusoidal Conditions}

In a nonsinusoidal, balanced three-phase system, the harmonics of the voltages and current phase waveforms can be divided into three groups with different sequence of phases. The set of harmonics of order $3 h+1$ (with $h=0,1$, $2, \ldots$ ) which have direct phase sequence, the set of order harmonics $3 h+2$, which are inverse phase sequence, and the set of harmonics $3 h+3$, which have homopolar phase sequence. However, in an unbalanced and nonsinusoidal system, each one of the voltage and current harmonics will have a direct sequence component, a inverse sequence component and a homopolar component.

Different components of the voltage at the PCC (point of common coupling) of a power system will be defined. Thus, deemed a component balanced, $\mathrm{Vb}$, constituted by the phasors of $3 \mathrm{~h}+1$ order harmonics of direct sequence, $3 \mathrm{~h} ` 2$ order harmonics of inverse sequence and $3 \mathrm{~h}+3$ order harmonics of zero sequence. The rms value of this balanced voltage is,

$$
V_{b}^{2}=\sum_{\forall h}\left[\left(V_{d}^{3 h+1}\right)^{2}+\left(V_{i}^{3 h+2}\right)^{2}+\frac{1}{1+\xi}\left(V_{0}^{3 h+3}\right)^{2}\right]
$$

where $\xi$ is taken equal to 1 in the Std. 1459, [5]. The terms $V_{d}, V_{i}$, and $V_{0}$, refer to the components of the direct sequence, inverse and homopolar, respectively, of the corresponding harmonics of the voltage.

The rest of the phasors of the symmetrical components of the harmonics are associated with the component of the unbalance voltage $V_{u}$, whose rms value is,

$$
\begin{aligned}
& V_{u}^{2}=\sum_{\forall h}\left[\left(V_{i}^{3 h+1}\right)^{2}+\frac{1}{1+\xi}\left(V_{0}^{3 h+1}\right)^{2}+\left(V_{d}^{3 h+2}\right)^{2}+\right. \\
& \left.+\frac{1}{1+\xi}\left(V_{0}^{3 h+2}\right)^{2}+\left(V_{d}^{3 h+3}\right)^{2}+\left(V_{i}^{3 h+3}\right)^{2}\right]
\end{aligned}
$$

In both rms, $V_{b}$ and $V_{u}$, is possible to extract the value for the fundamental harmonic. Thus, it is the fundamental balance component of the voltage, $V_{b l}$,

$$
V_{b 1}=V_{b}(h=1) \equiv V_{d}^{1}
$$

And the fundamental unbalance component, $V_{u l}$,

$$
V_{u 1}=V_{u}(h=1) \equiv \sqrt{\left(V_{i}^{1}\right)^{2}+\frac{1}{1+\xi}\left(V_{0}^{1}\right)^{2}}
$$

From the decomposition of the voltage in four components, is obtained the rms value of the voltage (effective voltage),

$$
V_{e}^{2}=V_{b 1}^{2}+V_{u 1}^{2}+V_{b H}^{2}+V_{u H}^{2}
$$

Similar decomposition is performed to the current, for example, the rms value of the balanced component of the current is,

$$
I_{b}^{2}=\sum_{\forall h}\left[\left(I_{d}^{3 h+1}\right)^{2}+\left(I_{i}^{3 h+2}\right)^{2}+(1+3 \rho)\left(I_{0}^{3 h+3}\right)^{2}\right]
$$

where $\rho$ is the ratio between the neutral conductor resistance and phase conductor resistance; in Std 1459, $\rho$ $=1$. Thus, the effective value of the current is,

$$
I_{e}^{2}=I_{b 1}^{2}+I_{u 1}^{2}+I_{b H}^{2}+I_{u H}^{2}
$$

The grouping of symmetrical to each set of harmonics components is viable from the viewpoint from the FFT of the Park vectors of voltage and current on one side, and the FFT of the zero sequence components, on the other. That is, for the determination of the components of direct and inverse sequence defines the current Park vectors,

$$
\mathbf{i}_{\mathbf{p}}=\sqrt{\frac{2}{3}}\left(i_{a}+a i_{b}+a^{2} i_{c}\right)
$$

where $a=\exp (\mathrm{j} 2 \pi / 3)$ and $i_{a}, i_{b}$ and $i_{c}$ are the phase currents. In the same way defining the Park vectors of the voltage.

The FFT of $\mathbf{i}_{\mathbf{p}}$ determines the direct sequence symmetrical components of each harmonic to the first half of the samples and the inverse sequence component to the second half,

$$
I_{F F T}(k)=\left\{\begin{array}{rlr}
I_{h}^{d} & \text { for } & k=h<\frac{N}{2} \\
\left(I_{h}^{i}\right)^{*} & \text { for } & k=N-h>\frac{N}{2}
\end{array}\right.
$$

In (9) is considered a set of N samples to the FFT of $\mathbf{i}_{\mathbf{p}}$. Since $k=1$ to $k=(N / 2)-1$ have symmetrical components of direct sequence of (N/2)-1 current harmonics. And from $\mathrm{k}=(\mathrm{N} / 2)+1$ to $\mathrm{k}=\mathrm{N}-1$ have the inverse sequence components conjugated of the same (N/2)-1 harmonic currents. Spectral analysis of the hompolar sequence components is performed through the FFT of actual samples of the homopolar component, [6]. A method identical to (8) and (9) is applied to the phase voltages. This original method for determining the phase sequence components not require the use of Fortescue transformation matrices as follows in [7-8].

\section{A New Apparent Power Decomposition}

The decomposition established in (5) and (7) to the RMS values of the voltage and current, respectively, allows to propose a partition of apparent power that fulfills the relation (10), 


$$
\begin{aligned}
& S_{e}^{2}=\left(3 V_{e} I_{e}\right)^{2}= \\
& =S_{b 1}^{2}+S_{u 1}^{2}+S_{b H}^{2}+S_{u H}^{2}
\end{aligned}
$$

which identifies the following four terms of power,

$$
\begin{gathered}
S_{b 1}^{2}=9 V_{b 1}^{2} I_{b 1}^{2} \\
S_{u 1}^{2}=9\left(V_{b 1}^{2} I_{u 1}^{2}+V_{u 1}^{2} I_{b 1}^{2}+V_{u 1}^{2} I_{u 1}^{2}\right) \\
S_{b H}^{2}=9\left(V_{b 1}^{2} I_{b H}^{2}+V_{b H}^{2} I_{b 1}^{2}+V_{b H}^{2} I_{b H}^{2}\right) \\
S_{u H}^{2}=S_{e}^{2}-S_{b 1}^{2}-S_{u 1}^{2}-S_{b H}^{2}
\end{gathered}
$$

$S_{b 1}$ is the balance fundamental component of apparent power, $S_{u l}$ is the unbalance fundamental apparent power, $S_{b H}$ is the balance harmonic apparent power, and $S_{u H}$ is the unbalance harmonic apparent power.

If taken $S_{b l}$ as the base value is possible to define indicators related to the unbalance and harmonic distortion in the form,

$$
\begin{gathered}
S U F=\frac{S_{u 1}}{S_{b 1}} \\
S B H=\frac{S_{b H}}{S_{b 1}} \\
S U H=\frac{S_{u H}}{S_{b 1}}
\end{gathered}
$$

SUF, SBH y $S U H$ are Unbalance Fundamental, Balance Harmonic, and Unbalance Harmonic factors of system, respectively. Similarly, is possible to define global indicators to assess the unbalance and the total harmonic distortion of the system according to the previous,

$$
\begin{aligned}
& S T H D=\sqrt{\frac{S B H^{2}+S U H^{2}}{1+S U F^{2}}} \\
& S T U D=\sqrt{\frac{S U F^{2}+S U H^{2}}{1+S B H^{2}}}
\end{aligned}
$$

STHD and STUD are Total Harmonic Distortion and Total Unbalance Distortion of System, respectively.

In the next section will determine the previous power terms and indexes for a practical case of nonsinusoidal and unbalanced power system.

\section{Experimental Results}

In the order to perform a validation of the proposal and verify the stability of the indices, has been built an experimental platform consists of three unbalance/nonlinear loads fed directly from the supply network, figure 1 . The load 1 is a balanced three-phase topology formed by three single-phase rectifiers with a RL branch into dc side. The load 2 is an unbalanced non-linear load constituted by a single-phase rectifier with a RC branch into dc side in one phase and two resistors of different values in the remaining phases. Finally, the load 3 is a linear inductive and unbalanced load.

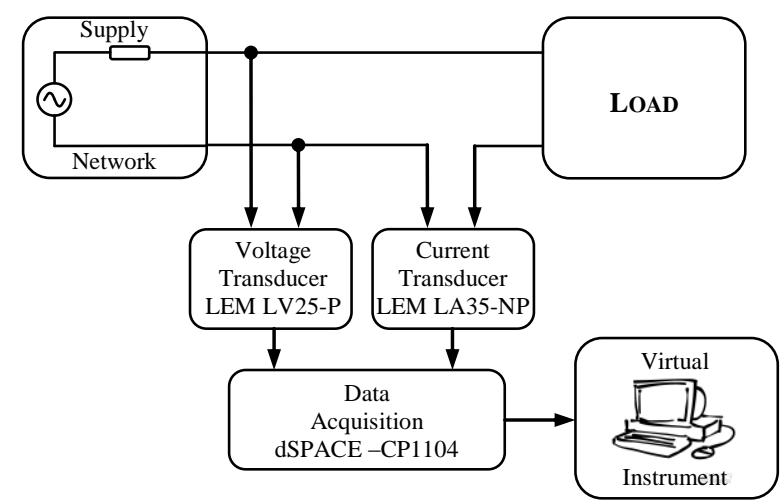

Fig. 1. Scheme of the test system on each phase

The measurement system consists of a data acquisition card (dspace-CP1104) and a signal conditioning system formed by three voltage sensors (LEM LV25-P) and three current sensors (LEM LA35-NP), thus the voltage and current signals are taken simultaneously, without introducing any phase change that could affect the accuracy of measurements. For data acquisition and processing, has developed a virtual instrument using Matlab and ControlDesk, see Figure 1. This instrument stores the instantaneous values of each phase voltage and line current. The configuration of the virtual instrument was made following the recommendations EN 61000-4-7 and EN 61000-4-30, so that has been used window equal to five cycles of the fundamental component and a sampling frequency of $6400 \mathrm{~Hz}$, thus avoiding problems of aliasing and leakage errors.

Table I presents the effective voltage value at the PCC, and the RMS values of the current at the terminals of the three loads. It also shows the value of apparent power of the set of three loads and the each individual load.

Table I. Effective Values of Voltage, Current, and Apparent Power

\begin{tabular}{|c|c|c|c|}
\hline & Ve (V) & Ie (A) & Se (VA) \\
\hline PCC & 229,69 & 58,10 & 40039,0 \\
\hline Load 1 & 229,69 & 23,19 & 15982,0 \\
\hline Load 2 & 229,69 & 13,69 & 9435,9 \\
\hline Load 3 & 229,69 & 35,10 & 24189,0 \\
\hline
\end{tabular}

Table II includes the results corresponding to the power terms of the three joint loads and each individual load according to the decomposition in (10).

Table II. Apparent Power Components in VA

\begin{tabular}{|c|r|r|r|r|}
\hline & \multicolumn{1}{|c|}{$\mathrm{S}_{\mathrm{b} 1}$} & \multicolumn{1}{c|}{$\mathrm{S}_{\mathrm{u} 1}$} & \multicolumn{1}{c|}{$\mathrm{S}_{\mathrm{bH}}$} & \multicolumn{1}{c|}{$\mathrm{S}_{\mathrm{uH}}$} \\
\hline PCC & 35514,0 & 15307,0 & 8244,4 & 6290,8 \\
\hline Load 1 & 12840,0 & 60,3 & 9510,1 & 329,3 \\
\hline Load 2 & 4262,3 & 2111,3 & 5130,3 & 6331,8 \\
\hline Load 3 & 18449,0 & 15639,0 & 310,7 & 307,8 \\
\hline
\end{tabular}


The results of table II show the high harmonic imbalance of the load 2 with respect to the load 1 and load 3 . On the other hand, load 1 presents a reduced value of the unbalance fundamental power and the unbalance harmonic power because it's a balanced load supplied from the PCC voltage. Load 3, linear and unbalanced, presents a high unbalance to the fundamental frequency and a small harmonic unbalance. This is synthesized in the indicators of unbalance and distortion introduced.

Table III presents the unbalance fundamental, SUF, balance harmonic, $\mathrm{SBH}$, and unbalance harmonic, $\mathrm{SUH}$, factors defined in (15), (16) and (17), respectively, in percentage values.

Table III. Unbalance and Harmonic Distortion Factors

\begin{tabular}{|c|c|c|c|}
\hline & SUF & SBH & SUH \\
\hline PCC & 43,10 & 23,21 & 17,71 \\
\hline Load 1 & 0,47 & 74,06 & 2,56 \\
\hline Load 2 & 49,53 & 120,37 & 148,55 \\
\hline Load 3 & 84,77 & 1,68 & 1,67 \\
\hline
\end{tabular}

The load 1 presents unbalance fundamental and unbalance harmonic values less than $1 \%$ and $3 \%$ respectively, as corresponds to a nonlinear balanced consumer. The load 2 on the contrary, shows values of about $50 \%$ and $150 \%$ for the same factors, specific to unbalanced nonlinear consumer. Load 3 has a high value of unbalance fundamental, about $85 \%$, and a value less than $2 \%$ of unbalance harmonic, specific of a linear unbalanced consumer.

Finally, Table IV includes the results of the assessment of the unbalance and overall distortion of the system in percentage values,

Table IV. Overall Harmonic and Unbalance Distortion

\begin{tabular}{|c|c|c|}
\hline & STHD & STUD \\
\hline PCC & 26,82 & 45,40 \\
\hline Load 1 & 74,11 & 2,09 \\
\hline Load 2 & 171,33 & 100,07 \\
\hline Load 3 & 1,81 & 84,77 \\
\hline
\end{tabular}

The first column identifies loads 1 and 2 as non-linear loads while the second column identifies 2 and 3 as unbalanced loads. The results presented in tables II, III and IV, extend those that could be obtained from the Std 1459. Thus, the latter will not allow determine the coming unbalance of harmonic distortion.

\section{Conclusión}

A new decomposition of the apparent power in the framework of Std. 1459 has been presented. This partition is based on a rigorous separation of the phase sequence components of the system harmonics. So, have been considered as balanced harmonics those of direct sequence of order $3 h+1$, of inverse sequence of order $3 h+2$ and homopolar sequence of order $3 \mathrm{~h}+3$. These harmonics are the balanced component of the voltage/current. The rest of sequence components of these harmonics form part of the unbalance component of voltage/current. The above classification is viable from the practical viewpoint through the use of the FFT Park vectors of voltage and current; this prevents unnecessary use of the transformation matrices of the symmetrical components for each of the harmonics.

The above treatment has allowed to propose a partition of apparent power in four terms of power: balance fundamental, unbalance fundamental, balance harmonic and unbalance harmonic of system. Through the above terms are defined three indicators of measures of the EPQ system: Unbalance Fundamental, Balance Harmonic and Unbalance Harmonic factors. In this way is possible to evaluate the unbalanced nonsinusoidal situations counting the true effects of the phenomenon of unbalance in the system. In the same way, these latter indicators were used to introduce two additional indices to assess the distortion and the total unbalance. The new partition of apparent power measures the unbalance introduced by harmonics. This is not possible using the Std-1459.

Finally, an experimental platform was developed to experimentally test the procedure, and the terms of power and indicators introduced. The results showed the viability of the proposed approach.

\section{Acknowledgement}

This work is part of the projects "Measurement System for the Identification of Harmonic Distortion Sources and Unbalances in Public Distribution Networks", ref. DPI2010-17709, sponsored by the "Ministerio de Economía y Competitividad" Government of Spain.

\section{References}

[1] IEEE Recommended practices and requirements for harmonic control its elect. power syst., IEEE Std. 519-1992.

[2] A. von Jouanne, and B. Banerjee, "Assesment of voltage unbalance", IEEE Trans. On Power Delivery, Vol. 16, No. 4, Oct 2001

[3] P. Pillay and M. Manyage, "Loss of life in induction machines operating with unbalanced supplies". IEEE Trans. Energy Conversion, Vol. 21, December 2006, pp. 813-822.

[4] T. E. Seiphetlho and A. P. J. Rens, "On the assessment of voltage unbalance", 14th International Conference on Harmonics and Quality of Power (ICHQP), 2010, pp. 1-6.

[5] "Definitions for the measurement of electric power quantities under sinusoidal, nonsinusoidal, balanced, or unbalanced conditions, IEEE Std. 1459-2000, January 2000.

[6] P. Salmerón and F.J. Alcántara, "A new technique for unbalanced current and voltage measurement with neural networks" IEEE Trans. on Power Delivery, Vol. 20, No. 2, Mayo 2005, pp. 852-858.

[7] T. Zheng, E.B. Makram, A.A. Girgis, "Evaluating power system unbalance in the presence of harmonic distortion". IEEE Trans. on Power Delivery, Vol. 18, No. 2, April 2003, pp. 393397

[8] G. Chicco, P. Postolache, and C. Toader, "Analysis of threephase systems with neutral under distorted and unbalanced conditions in the symmetrical component-based framework" IEEE Trans. on Power Delivery, Vol. 22, No. 1, January 2007, pp. 674-683. 